\title{
CASE OF A WOUND
}

OF THR

\section{PERONEAL ARTERY,}

SUCCESSFULLY TREATED BY LIGATURE.

BY GEORGE JAMES GUTHRIE, EsQ.

Read May 14, 1816.

THE surgery of diseased and wounded arteries has within these few years been so much improved by the science and true professional boldness of several of our best surgeons, that little perhaps remains to be added to the principles on which operations have been instituted on the more important vessels of the human body. The external and internal iliac, the carotid, the femoral, and the axillary arteries have all been secured by the surgeon, and the ligature of the subclavian has been frequently, although hitherto unsuccessfully, attempted*. The operations to be performed on these

* Since the Ist edition of this work was published, the subclavian bas been twice tied successfully. Once in America, and once in Paris. 
large vessels have occupied the attention of every surgeon, and the necessity for their performance frequently occurring, both from circumstances of disease and accident, has rendered them objects of more universal interest to the junior branches of the profession, than those on the smaller vessels, which from their being less exposed to accident, less liable to bad consequences after injuries, and not so immediately the seat of operations from disease, have not made so strong an impression, and are not so much attended to as they perhaps deserve. I allude especially to the three arteries of the leg, and with this impression, I beg now to claim the indulgence of the Society for the relation of a case, in which the peroneal artery was successfully tied. I would not trespass on the time of the Society with a case that may be considered by many so unimportant, if I could find any other satisfactorily recorded, in which the operation of securing it by ligature in the thick part of the calf of the leg had been successfully accomplished. I am aware that this operation must very seldom indeed be necessary from disease, and the artery is well protected against all the common accidents of domestic life; but as every part is exposed in military warfare, I am inclined to believe this has not hitherto escaped, but that operations have been performed in consequence, which I consider improper, or that nature has been able by the exertion of her own powers to prevent hæmorrhage, as we know fre- 
quently occurs on the division of the femoral or brachial arteries, and I believe even in one case of the external iliac.

The subject of my operation, Henry Vigaribe, a private in the German legion, was wounded on the 18th of June, at the battle of Waterloo, by a musket ball which entered the right leg immediately behind and below the inner head of the tibia, inclining downwards, and under or before a part of the soleus and gastrocnemius muscles, and com. ing out through them four inches and three quarters below the head of the fibula, nearly in the middle, but towards, the side of the calf of the leg. In this course it is evident that the ball must have passed close to the posterior tibial and peroneal arteries; but as little inflammation followed, and no immediate hæmorrhage, he was considered to be one of the slighter cases. On the latter days of June, he occasionally lost a little blood from the wound, and on the 1st of July a considerable hæmorrhage took place, which was suppressed by the tourniquet, and did not immediately recur on its removal. It bled, however, at intervals during the night; and on the morning of the $2 \mathrm{~d}$, it became necessary to re-apply the tourniquet, and to adopt some means for his permanent relief.

Having gone to Brussels after the battle of $\mathrm{Wa}$ terloo, with the view of obtaining additional sup. 
port to some opinions I had advanced in military surgery, this man was shewn to me by my friends, Messrs. Campbell and Hill, surgeons to the forces, under whose care he was, and who were desirous of avoiding an amputation, if possible, although the site of the wound, and the uncertainty of the vessel wounded, as it bled from both openings, rendered it doubtful.

The man had lost a large quantity of blood from the whole of the bleedings, his pulse was 110 , the skin hot, tongue furred, with great anxiety of countenance : the limb, from the application of the tourniquet from time to time, was swelled, a quantity of coagulated blood had forced itself under the soleus in the course of the muscles, increasing the size of the leg, and florid blood issued from both openings on taking the compression off the femoral artery. On passing the finger into the outer opening, and pressing it against the fibula, a sort of small aneurismal tumour could be felt under it, and the hæmorrhage ceased, indicating that the peroneal artery was in all probability the only vessel wounded.

In this case there was, in addition to the wound of the artery, a quantity of blood between the muscles, which in gun-shot wounds accompanied by inflammation is always a dangerous occurrence, as it terminates in profuse suppuration of the con. 
taining parts, and frequently in gangrene. The evacuation of it, therefore, became an important consideration, even if the hæmorrhage had ceased spontaneously.

The man being laid on his face with the calf of the leg uppermost, I made an incision near seven inches in length in the axis of the limb, taking the shot-hole nearly as a central point, and carried it by successive strokes through the gastrocnemius and soleus muscles towards the peroneal artery, which I attempted to discover, but this was more difficult than might be supposed, after such an opening had been made. The parts were not easily separated, from the inflammation that had taken place, and those in the immediate track of the ball were in the differing stages from sphacelus to a state of health, as the ball in its course had produced its effect upon them, or their powers of life were equal or unequal to the injury sustained.

The sloughing matter mixed with coagulated blood readily yielded to the back of the knife, but was not easily dissected out. The spot where the arterial blood came from was distinguishable through it, but the artery could not be perceived, the depth of the wound rendering any operation on it difficult. To obviate this inconvenience, I made a transverse incision outwards from the shothole to the edge of the fibula, which enabled me to 
turn back two little flaps; and gave greater facility in the use of my instruments. I could now pass a tenaculum under the spot from whence the blood came, which I raised a little with it, but could not distinctly see the wounded artery in the altered state of parts so as to secure it separately. I therefare passed a small needle, bearing two threads, a sufficient distance above the tenaculum, to induce me to believe it was in sound parts, but including very little in the ligature, when the hæmorrhage ceased: another was passed in the same manner below, and the tenaculum withdrawn. The coagula under the soleus were removed, the cavity washed out by a stream of warm water injected through the external opening, the wound gently drawn together by two or three straps of adhesive plaster, and the limb enveloped in cloths constantly wetted with cold water. The patient was placed on milk diet.

On the 4th, two days after the operation, the wound was dressed and looked well, the weather being very hot, and two straps of plaster only applied to prevent the parts separating. On the 5 th, a poultice was laid over the dressings, in lieu of the cold water, the stiffness becoming disagreeable. On the 6th, as the wound, although open in all its extent, did not appear likely to separate more, the plasters were omitted, and a poultice alone applied. On the 8 th and 9 th, it 
suppurated kindly; and on the 10th'; or eight days from the operation, the ligatures came away, the limb being free from tension, and the patient in an amended state of health, in consequence of his medical treatment having been steadily attended to.

From this period, the cure went on, although slowly, without accident; a small abscess formed at the inner and lower edge of the soleus muscle, but closed shortly after its contents were evacuated. The wound was entirely healed in three months, but the leg was bent on the thigh, and required mechanical means for its extension.

The length of the fibula is 16 inches. The cicatrix of the wound made by the ball is $4 \frac{3}{4}$ inches, below the head of the fibula. The sound limb, $4 \frac{3}{4}$ inches from the head of the fibula, is $13 \frac{3}{4}$ inches in circumference. The limb operated on $11 \frac{3}{4}$ inches, being a diminution of two inches. The length of the cicatrix is $6 \frac{I}{2}$ inches. The artery was tied therefore by computation $1 \frac{1}{4}$ inches below where it is usually given off by the posterior tibial.

The man is now in the York Hospital at Chelsea, and walks about without appearing lame, although he cannot do so for any great distance. He suffers no pain, except an occasional kind of cramp in the ball of the foot, and some contraction 
332 CA8E OF WOUNDED PERONEAL ARTERY. of the toes, which takes place generally when he rises in a morning, and continues for a minute or two, until he puts them straight with his hand, which I do not attribute to the operation, but to some additional injury done to the nerves by the ball in its course through the leg. 